\title{
Cheating in a dental practical exam
}

\author{
Wendy Currie $^{1 *}$ (D) Susie Dracopoulos ${ }^{2}$ and Graham Hendry ${ }^{3}$
}

\author{
* Correspondence: \\ wendy.currie@sydney.edu.au; \\ http://sydney.edu.au/dentistry \\ ${ }^{1}$ Faculty of Dentistry, The University \\ of Sydney, 1 Mons Rd Westmead, \\ Sydney, NSW 2145, Australia \\ Full list of author information is \\ available at the end of the article
}

\begin{abstract}
There is increasing attention given to academic integrity across university education and dental schools are not immune to this problem (Andrews et al. J Dent Educ 71; 1027-1039, 2007; Ford \& Hughes Eur J Dent Educ 16(1):e180-e186, 2012). While there has been an increasing concern about academic dishonesty in written exams and assignments, there appears to be a false sense of security in the integrity of practical assessments, involving dental procedures on simulated patients.

This paper will present a situational analysis of two unusual cases of academic dishonesty in preclinical dental practical assessments. The first case involved a student bringing a previously prepared plastic tooth into the examination room and substituting it for the assessment tooth. The second case involved a student removing key teeth during the exam to enable them to have better access and advantage over others to complete the assessment task. These two cases resulted in a complete review of practical assessment procedures and the application of new processes to maintain academic integrity.
\end{abstract}

Keywords: Dental students, Dental practical exams, Cheating, Dishonesty, Academic integrity

\section{Introduction}

The range of assessment types used in a dental program is wide and includes direct observation of patient treatment and practical simulation assessments, as well as written examinations and other individual and group assessments. This ensures that students will demonstrate attainment of the expected graduate attributes and competencies of professionalism, communication and social skills, scientific and clinical knowledge and patient care (Australian Dental Council, 2016).

The practical pre-clinical assessments in our dental program take place in a simulation clinic equipped with mannequins housing plastic teeth. This provides an authentic learning environment to encourage the successful application of clinical knowledge and performance of skills in patient care (Driscoll, 2005). In keeping with the context, the practical dental assessments are authentic assessments - that is, assessments designed to replicate the future work requirements of a practising dentist. These assessments occur in the disciplines that have a practical element and include Tooth Conservation, Endodontics, Prosthodontics, Radiology, Periodontics, Local Anaesthesia and Exodontia. Furthermore, these authentic assessments are critical in enabling faculty to judge the student's aptitude for performing in the clinical setting and thus determine the student's competence (Yip \& Smales, 2000).

(c) The Author(s). 2017 Open Access This article is distributed under the terms of the Creative Commons Attribution 4.0 International License (http://creativecommons.org/licenses/by/4.0/), which permits unrestricted use, distribution, and reproduction in any medium, provided you give appropriate credit to the original author(s) and the source, provide a link to the Creative Commons license, and indicate if changes were made. 
In the past, the Faculty had been operating under the assumption that cheating in a practical exam was highly unlikely, if not impossible. Indeed, the Faculty was aware of only a very small number of academic integrity breaches in past years, and as recently as 2013 there were no cases of academic dishonesty reported to the University's Academic Board.

However, more recently in 2014, the Faculty upheld 8 cases of plagiarism and academic dishonesty. This number represented $0.83 \%$ of Postgraduate Dental Students and 3.03\% of Undergraduate Dental Students. The dishonest behaviors occurred in the first semester and included a group communicating during a written exam, students colluding to reproduce written exam answers for following cohorts, students colluding on individual assignments, and most unusually two distinctly different counts of students cheating during a practical examination.

The significant spike between the 2013 academic year and the subsequent year were of great concern to the Faculty, particularly the breaches in 2014 within the practical examination - a preclinical practical exam of the type common to many dental schools. There had been a general sense of confidence that authentic practical assessments were secure from breaches of academic integrity. In fact, the application of assessment methods, which are clearly connected to clinical practice, are thought to be perceived by the student as being relevant and therefore the tendency to cheat is minimised (Warman et al., 1994; Aaron et al., 2011).

There was also an underlying assumption made by our academics that students would understand the significance of a preclinical practical exam. Of primary concern to the Faculty was that these breaches of clinical integrity could ultimately compromise patient care and safety (Lingen, 2006).

The students involved were both in the third year of a four-year postgraduate professional Master's Degree, the Doctor of Dental Medicine. A review of their academic record revealed that they were marginal to average students, who at times had required remediation and reassessment for similar practical exams, but, they were both on track to meet the requirements of progression based on their continuous clinical and simulated clinical practice. We were unable to understand why they cheated, primarily because both students refused to admit that they cheated, even when presented with irrefutable evidence of their breaches of integrity.

This paper aims to present these two unusual cases of academic dishonesty. It will describe the framework for the situational analysis and the processes undertaken for identifying how they occurred and were addressed. In conclusion, we will put forward suggestions for best practice in ensuring educational integrity in dental practical exams.

\section{Background}

In 2014, the third-year dental student cohort was tasked with preparing a crown preparation of an upper molar plastic tooth in a typodont model during their preclinical practical exam. This assessment task required students to perform all the steps and procedures that would be performed on a real patient in a dental clinic. It also needed to be performed within a clinically acceptable timeframe, which directly related to realistic expectations of patient tolerance. This was a high stakes assessment, which served as a progression barrier. 


\section{Case A}

During the exam, student $\mathrm{X}$ was displaying several unusual behaviors, which included: retrieving the exam tooth and turning it upside down to inspect the black identifying markings on the underside. During set up time, the student left the exam room to allegedly retrieve safety glasses. Throughout the exam the student appeared to be doing less cutting compared to other students, was handling a screwdriver during the exam and appeared to conceal items on their instrument tray. At the completion of the exam, students were asked to unscrew their exam tooth and tape it to their exam paper for submission. Tutors looked at the prepared tooth of student $\mathrm{X}$ and noticed that the black marker ink was wet and easily displaced when touched (Fig. 1). This was in contrast with the other assessment teeth submitted by other students.

The case was reviewed by an academic panel who found that, despite denial by the student, cheating had occurred by submitting a previously prepared tooth in place of the examination tooth. The required competence in performing under the simulated clinical conditions could not be determined and as a result, the student failed the assessment task and would be required to sit a reassessment. This meant that the maximum grade attainable would be at a pass level. Additionally, the student was required to write a reflection report on the importance of integrity in Dentistry.

\section{Case B}

The same exam was repeated in the afternoon session for the other half of the cohort. Towards the end of the exam, student $Y$ was observed to have a missing adjacent tooth. Figure 2 shows the student was preparing the upper left first molar tooth but the upper left second molar tooth was missing allowing student $Y$ easy access and an unfair advantage over other students.

When questioned by the tutor why the adjacent tooth was missing the student replied it became dislodged and fell to the back of the simulated patient's mouth and had entered the suction system. The tutor asked why the exam tooth was loose, as students had been advised to have all teeth present and secure at the start of the exam. The student replied that they did not have any more screws and often used wax to secure plastic teeth in the typodont model.

This case was also reviewed by an academic panel who found that the student had, gained an unfair advantage over other students. By having the adjacent tooth missing, the student had enhanced access and vision that would not be found with patients with a full set of teeth. The panel determined that the required competence in performing

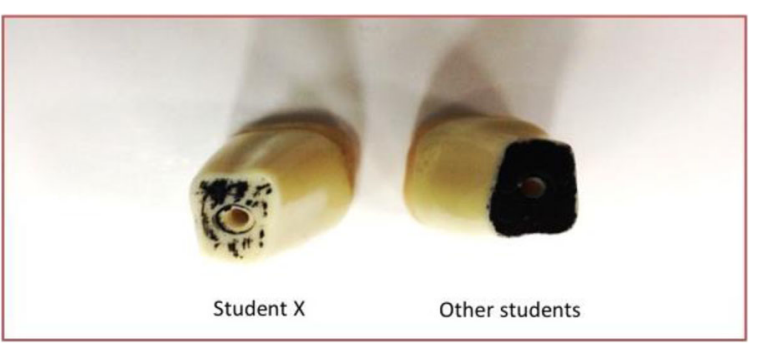

Fig. 1 Prepared exam teeth submitted for marking 


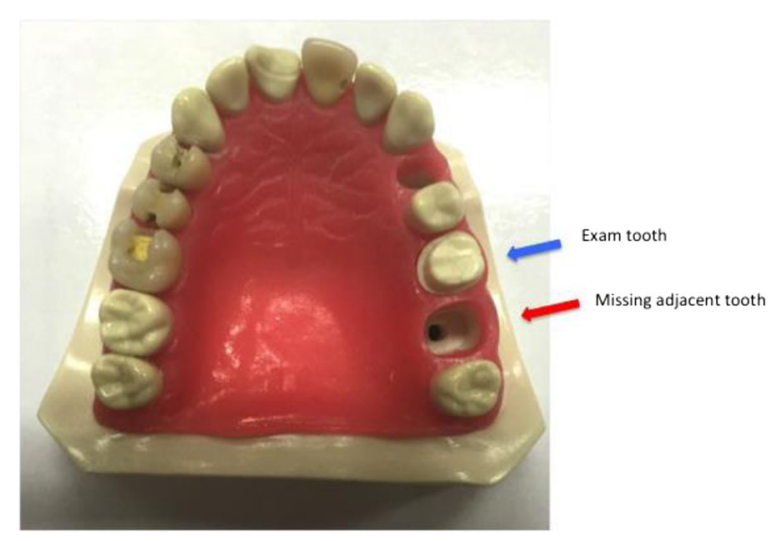

Fig. 2 Typodont with missing adjacent tooth

under the simulated clinical conditions could not be determined and as a result, this student also failed the assessment task and was required to reassess and submit a reflection report addressing the same topic as the student in Case A.

\section{Literature review}

A search of the literature using Ovid Medline returned results which revealed that the literature specifically concerning academic integrity in dental and oral health programs is relatively very small, but it highlights cheating and academic dishonesty as a problem for dental schools (Andrews et al., 2007; Ford and Hughes, 2012).

The search revealed 5472 articles pertaining to dental students. Given that there are over seven hundred dental schools identified on the World Dental Schools List (http:// www.dentaljuce.com/fruit/docs/World_Dental_schools.htm), this highlights that the search commenced with a very small foundation. Refinement with key word searches: 'dental students' 'cheat' and 'dishonest' resulted in only 36 articles.

The key focus in the literature was on perceptions, including students' perceptions of the assessment environment (Quick, 2014) and methods to control cheating (Escudier et al., 2014). Several studies have also examined dental students' perceptions of the extent of deception and cheating in their courses (Kumwenda et al., 2013; Fuller \& Killip, 1979; Lancaster et al., 1989), the relative severity of cheating behaviours (Al-Dwairi and Al-Waheidi, 2004), and the stress students experience in assessments (Kumar et al., 2009). Several articles focused on the attitudes and perceptions of both faculty staff and students to academic dishonesty and the penalties for cheating (Koletsi-Kounari et al., 2011; Asokan et al., 2013; Teplitsky, 2002) and one study investigated methods for enhancing students' ethical behaviour (Koerber et al., 2005), including the formulation of honour codes to maintain academic integrity (Turner \& Beemsterboer, 2003). Two studies sought the perspective of Deans in relation to policy and procedures to prevent cheating (Graham et al., 2016) and the incidence of cheating within their dental school or faculty (Beemsterboer et al., 2000).

In relation to the incidence of cheating by dental students, there is wide variance in the literature. In a study by Fuller and Killip (1979), 43\% of dental students admitted to cheating, while $94 \%$ believed that it occurred. In a later study (Warman et al., 1994), $30 \%$ of the dental students acknowledged that cheating during exams is a common 
practice. Alarmingly, one survey (Muhney et al., 2008) found that $86.5 \%$ of graduating Texas Dental Hygiene students admitted to having cheated at least once during their candidature.

The search terms were further broadened to include 'students/dental,' 'cheat,' 'dishonest' and 'practical exam'. Interestingly, this returned a result of zero. The fact that we could not locate any studies that have investigated the actual incidence of and potential for student cheating and academic dishonesty to occur in a dental practical assessment was surprising. This highlights a significant knowledge gap in this area.

\section{Methodology}

As the academic integrity breaches occurred within a clinically relevant assessment, this indicated the need for closer investigation of the assessment processes. A working group consisting of the Faculty Chair of Assessment, the Associate Dean of Learning and Teaching and members of the Assessment Committee was established to develop the framework for the situational analysis of the integrity breaches, which involved:

1. Identification and analysis of the internal and external elements within the current practical assessment process which could potentially impact on academic integrity

2. Development and communication of the new processes to better support educational integrity in dental practical exams

3. Evaluation of the intervention strategy

\section{Findings}

Identification of the elements

Practical examinations like this assessment are conducted in many disciplines of dental programs, including Tooth Conservation, Endodontics, Prosthodontics, Radiology, Periodontics, Local Anaesthesia and Exodontia. Therefore, an opinion survey was distributed to the Academic Head of each discipline. The survey focused on the rules that were applied to their practical exams and how they maintained academic honesty.

From the results of the survey, it was found that each discipline had different expectations for student conduct during practical assessments. There was inconsistency in the verbal instructions given at the start of the exam, ranging from explicit directions for some assessments to no instructions or rules provided for students in other instances. The tutor to student invigilation ratios were inconsistent and in many cases, there were an insufficient number of tutors to effectively oversee large groups of students. Many disciplines would inform the students which tooth and preparation type would be examined, to reduce student anxiety about the assessment task, and permit students to come to the assessment with appropriate adjacent teeth in their models. While this was viewed by the tutor as an efficient way to manage the assessment, it provided opportunity for acts of dishonesty. It was also discovered that in some assessments the students were permitted to access their complete 'dental toolbox' in the examination room, which meant they had access to screwdrivers that could be used to remove or replace plastic teeth. Toolboxes also provided a place to conceal items such as additional plastic teeth or instruments. 
There were also major issues identified in relation to the security of the examination room, meaning that the simulation clinics could potentially be accessed by students in the lead up to the exam. Tutors had also been using a method of identifying assessment teeth from practice teeth consistently over many years (by using black permanent ink markings), which meant that students would be aware of how to mark any tooth and submit it as their assessment tooth.

While tutors stated that they were comfortable with the university requirements for academic integrity in other standard (particularly written) forms of assessment, many indicated confusion when it came to practical exams. They felt that the policies did not relate to the specifics of a dental practical exam and stated that they did not know how to address a suspected breach of academic integrity.

This pointed to two key factors that enabled cheating. Firstly, there were inadequate policies and procedures in place. Secondly, it appeared that students were lax in their perception of exam conditions in practical exams and staff too where unaware of detecting cheating, how to avoid it, and what to do when it was observed.

While the University had policies related to maintaining academic integrity within standard types of written assessments, they did not cover the unique issues within the dental practical assessment. This concept has been the subject of a report led by the University of Newcastle and supported by a grant from the Australian Office for Learning and Teaching (OLT) (Commonwealth of Australia, 2016), which identified that the definitions of academic integrity for written assessment items do not apply in the same way for assessments not involving text. Further, the report recommended that where non-textual assessment items are used, the disciplines must define the specific parameters of academic integrity.

\section{Development and communication}

The first phase of the intervention in our dental program was the development of clear procedural guidelines for staff and students during practical exams. These were developed by the Working Group and included best practice elements from existing dental board examinations (Australian Dental Council, 2015; National Dental Examination Board of Canada, 2017) with links to University academic integrity policies. These guidelines were encompassed in a comprehensive document that addressed the unique aspects of dental practical assessments, and the document was distributed to all students and tutors.

The document provided a clear definition of educational integrity in dental practical assessments, citing examples of behaviours that would be considered breaches. It also clarified the specific process for dealing with a suspected case of academic dishonesty and included a suggested script to be delivered at the commencement of practical exams (Fig. 3), which reminded students of their responsibilities in relation to academic integrity in a dental practical exam.

General assessment guidelines were stated, such as dealing with emergency evacuations, student absences and the process for dealing with students unable to complete the assessment due to sickness. Standardised rules in relation to entering and leaving the exam room and the application of allocated seating were also included. 
You are not permitted to bring any unauthorised equipment or materials into this room. Please hand in any unauthorised equipment or materials to the invigilator or examiner. From this point, any unauthorised material or equipment will be confiscated. Please note that this is a confidential exam and as such no copies of the exam paper or questions are to be removed from this room at the end of the exam.

You are reminded not to engage in academic dishonesty during this exam. Academic dishonesty means seeking to obtain or obtaining academic advantage by dishonest or unfair means or knowingly assisting another student to do so. In practical exams, this includes, but is not limited to:

- Engaging another person to sit your exam

- Communicating by speaking or any other means during the exam (exception when seeking assistance to cure)

- Bringing unauthorised material or equipment into the exam room

- Attempting to gain enhanced access by removing any part of the mannequin or working on an incomplete mannequin

- Accessing your toolbox during the assessment

- Using a screwdriver during the assessment

- Copying from another student during the exam

- Inappropriately using electronic equipment during the exam

(NOTE: this list should be modified to suit the specific practical exam)

Students who engage in academic dishonesty during the exam will be subject to a report which may lead to misconduct proceedings.

If you need to leave the exam room during the exam, please raise your hand and notify the invigilator or examiner. Note that you are not permitted to leave the exam room and return during set up time and within the first 30 minutes of the exam.

The examiner will then state information specific to the exam:

You are sitting the DMD1 FCDA Tooth Conservation Summative Clinical Competency Assessment. It consists of one page. Please check that your paper is complete and raise your hand if it is not.

This exam is (2.5 hours duration) long and will stop at (11.30/3.30) according to the clock on screen. You may now start.

Fig. 3 Suggested exam script

The specialised elements of dental practical assessments were addressed, including a clear definition of permitted and prohibited equipment and materials; specified time frames for setting up prior to the assessment; and the dress requirements related to personal protection and infection control.

Phase two of the intervention involved the use of unique specification and identification of exam teeth. Changing the colour of the permanent marker between sessions was the simplest method; however, after positive discussions with the manufacturer of typodont teeth we arranged to have a customised stamp applied to each exam tooth, Fig. 4. This ensured the unique identification of the exam tooth was indeed different every time.

In 2011, the faculty Academic Simulation Coordinator together with the supplier of tooth models (One Dental) implemented an Australian first tooth vending machine to supply replacement plastic teeth for student models. These types of dispensing machines can now be found nationally in Australian Dental Schools. One Dental, is now fielding international inquiries in relation to applying this process for dispensing plastic teeth and dental equipment. In 2014, we approached the supplier and manufacturer with our cheating issue, which resulted in the development of unique stamping identification and accompanying operating procedure. The supplier/manufacturer reports that this idea has now been adopted for use in Australian national accreditation examinations.

Previously, many disciplines would inform the students which tooth and preparation type would be examined, to permit them come to the assessment having already purchased the plastic exam teeth. A student behaving unscrupulously, such as 


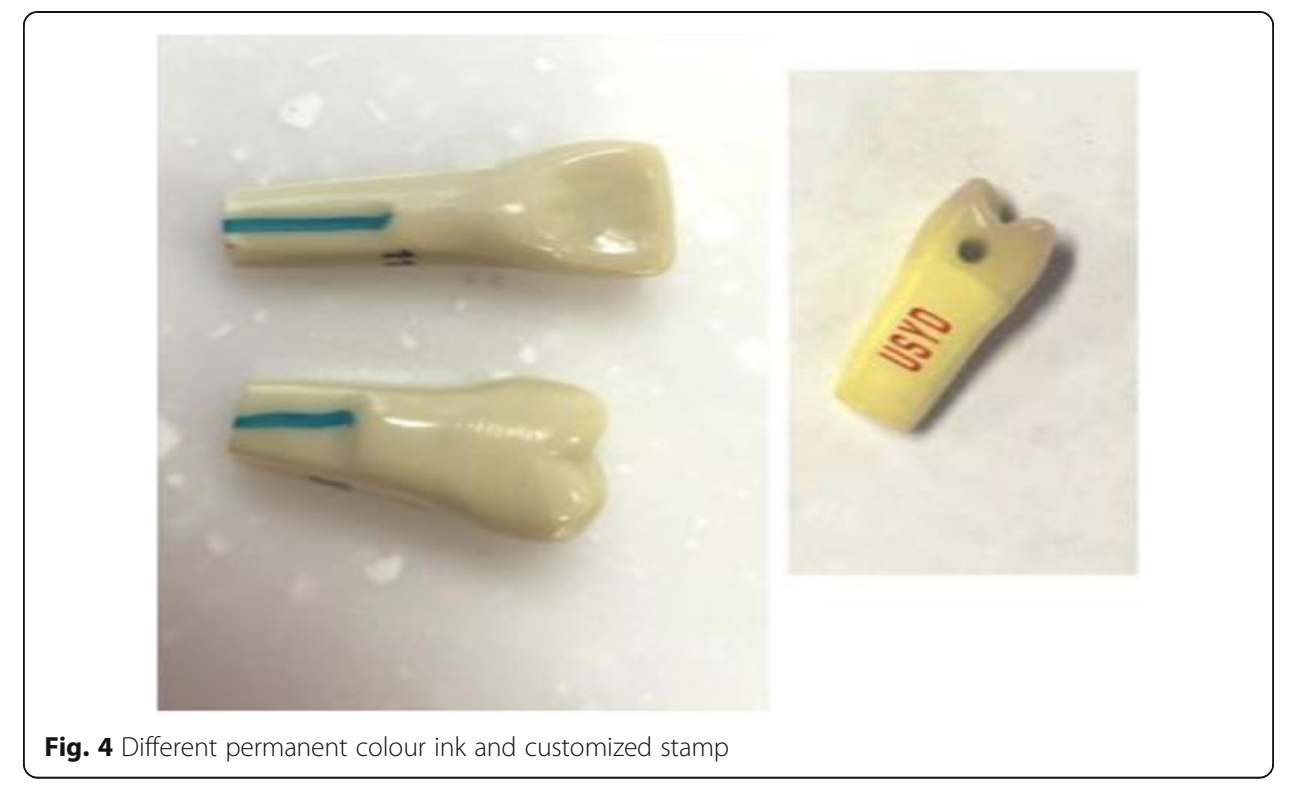

student X, could prepare several teeth in advance to substitute for the exam tooth. Under the new guidelines, the students would not be given this information, and a vending machine (Fig. 5) in the exam room, would contain a token that was exchanged for the exam tooth/teeth only after the students had arrived in the exam room. Again, this reinforced the unknown elements of dental clinical practice and assisted in maintaining the equity and integrity of the assessment task. Additionally, exam tasks were not repeated on the same day and different teeth had to be selected for morning and afternoon sessions.

The new procedures and processes were developed to address the issues above and were reviewed and revised by The Faculty Assessment Committee and each of the relevant disciplines. Consultation and approval was also sought through the student association. The final guidelines were presented to the Learning and Teaching Committee for ratification and communication to faculty and student representatives.
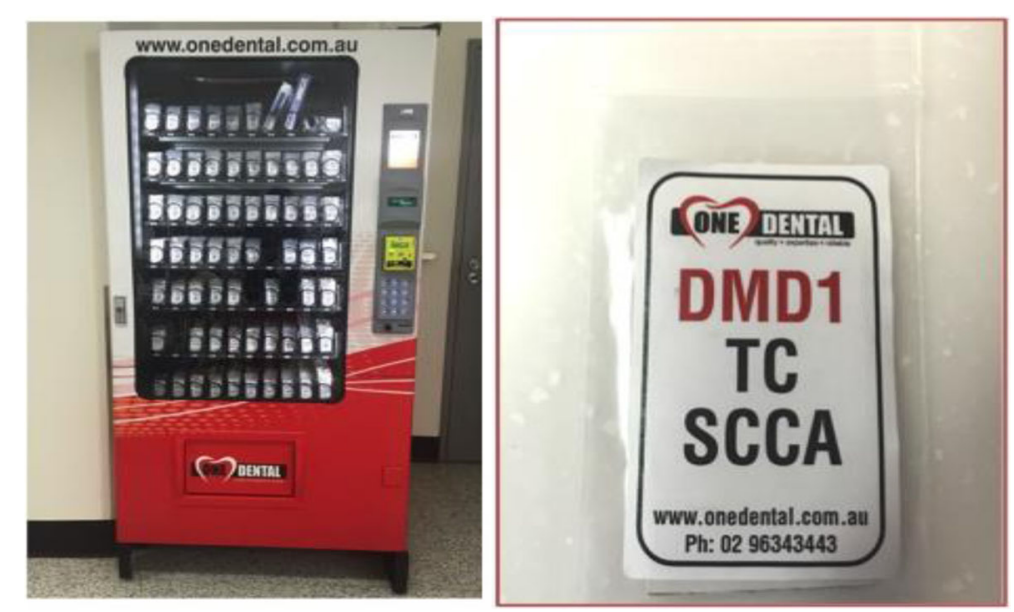

Fig. 5 One Dental vending machine and token 
The practical exam guidelines and processes in the Dental Faculty were enacted in the second semester of 2014. To evaluate the effectiveness of the interventions, a standing agenda item on academic integrity was added to the Assessment Committee Meeting following each exam period. This allowed for regular discussion between the key academics and professional staff involved with assessments.

The semester one 2015 review revealed that there had been 100\% uptake of the new processes. Invigilators reported that they had found the processes helpful and increased their confidence in maintaining academic honesty. The review of the second semester 2015 showed that a few of the disciplines were not adhering strictly to the guidelines by reverting to some of their bespoke processes. This indicated a need for vigilance in regularly making staff aware of the standardised processes and their responsibility for adherence. As a result, the Academic Head of each discipline is required to brief all staff involved with invigilation prior to each exam period.

Due to changes in the overarching university policies related to academic honesty during 2016, the review identified minor malalignment, particularly in relation to terminology and management. This resulted in administrative amendments of the guidelines and highlighted the need for regular reviews and communication with the invigilators involved with assessment.

Of prime interest is the fact that there have been no detected cases of academic dishonesty in practical exams in 2015, 2016 and semester 1 2017. Does this mean the new processes have been effective or have the students simply found smarter ways to cheat? It is our opinion that ongoing, regular evaluation is critical for detecting not only the known cases of academic dishonesty, but also identifying potential threats to the integrity of the dental practical exam.

\section{Discussion}

On reflecting our experience, we put forward the following observations and recommendations:

Firstly, in our search of the literature, we were unable to find any research specifically relating to cheating in a dental practical exam. This could indicate that our experiences are isolated and may not be representative of dental practical exams in other dental schools; however, we believe that our previous false assumption, that the dental practical exam was secure from breaches of academic integrity could be held by many dental faculties. We suggest that there is a significant gap in the literature and a pressing need for further research to be conducted in this area.

Secondly, we discovered that all assessment types are susceptible to breaches of academic integrity. It is naïve to think that any assessment format is secure from integrity breaches. Regardless of whether the task is authentic and requires the student to perform a procedure, a student behaving unscrupulously will find ways to cheat (Fuller \& Killip, 1979) Faculty should closely examine their assessment tasks and processes to attempt to detect any elements of risk.

Any program where non-standard assessment types are used will require unique guidelines to uphold academic integrity. University academic integrity policies generally deal well with standard written exams, but often do not cover the specific aspects, such as those found in dental practical exams. Clinical procedure guidelines, which focus on maintaining academic integrity, are necessary. Faculties should develop standardised 
processes relevant to their area (Asokan et al., 2013). Staff must be aware of the guidelines and policies and be frequently reminded of their responsibility for implementation. This will in turn promote an environment of professionalism and ethical behavior. It may also be worthwhile to turn to industry partners for solutions.

It is important to be cognisant of the vast difference in perceptions of academic integrity between staff and students (Ford \& Hughes, 2011; Koletsi-Kounari et al., 2011; Hutchins \& Cobb, 2008). Students may focus on 'jumping the hurdle' and may not consider the deeper implications, that is, progression to patient care. By explicitly highlighting what constitutes cheating in practical dental exams, students should develop a better understanding of academic integrity.

\section{Conclusion}

In conclusion, research has found a positive correlation between cheating in medical school and dishonesty in the care of patients (Sierles et al., 1980). Given the similarities between the medical and dental professions, it is expected that this correlation would also be true for dentistry (Lingen, 2006). Therefore, being aware of academic integrity, particularly in an authentic dental practical assessment should be of paramount concern for dental faculties. The integrity of the dental faculty, the dental profession and most importantly, the safety and the oral health of patients could be at risk. While this paper focused on integrity breaches in a dental practical exam, we suggest that this should be a strong consideration for any faculty using practical assessments, particularly where the graduate will be responsible for the ethical care of a patient.

Faculty must remain vigilant in relation to all assessments. A crucial factor in our experience was that we made several naïve assumptions. Firstly, we assumed that it was not possible to cheat in a dental practical exam. Secondly, we assumed that staff were applying sound processes and providing a secure environment while conducting these exams. By focusing on and addressing the issue of educational integrity, Dental Schools can actively model ethical behavior and positively impact the development of students' professionalism.

Authors' contributions

The original concept was developed by WC. SD and WC collected case study data and wrote the article. GH contributed to writing and editing of the article. All authors read and approved the final manuscript.

Competing interests

The authors declare that they have no competing interests.

\section{Publisher's Note}

Springer Nature remains neutral with regard to jurisdictional claims in published maps and institutional affiliations.

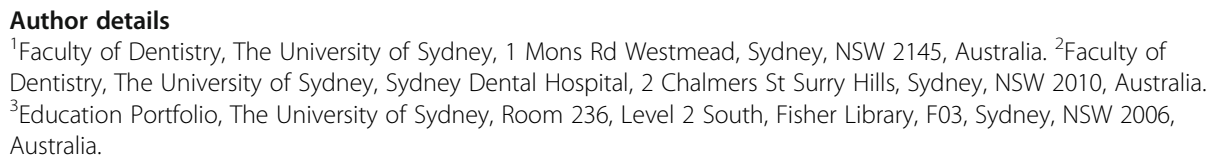


Asokan S, John JB, Janani D, Jessy P, Kavya S, Sharma K (2013) Attitudes of students and teachers on cheating behaviors: descriptive cross-sectional study at six dental colleges in India. J Dent Educ 77(10):1379-1383

Australian Dental Council. (2016). Professional Competencies of the Newly Qualified Dentist Retrieved from http://www. adc.org.au/documents/Professional\%20Competencies\%20of\%20the\%20Newly\%20Qualified\%20Dentist\%20\%20February\%202016.pdf

Australian Dental Council Ltd. (2015). Australian Dental Council Practical Examination Handbook Retrieved from http:// www.adc.org.au/documents/Practical\%20Examination\%20Handbook\%202015.pdf

Beemsterboer PL, Odom JG, Pate TD, Haden NK (2000) Issues of academic integrity in U.S. dental schools. J Dent Educ 64(12):833-838

Commonwealth of Australia (2016) Plagiarism and related issues in assessments not involving text. Australian Government Office for Learning and Teaching, Sudney, Australia Retrieved from http://apo.org.au/node/65099

Driscoll MP (2005) Psychology of learning for instruction, 3rd edn. Pearson Allyn and Bacon, Boston

Escudier MP, Tricio JA, Odell EW (2014) Student acceptability of high-stakes e-assessment in dental education: using privacy screen filters to control cheating. J Dent Educ 78(4):558-566

Ford PJ, Hughes C (2012) Academic integrity and plagiarism: perceptions and experience of staff and students in a school of dentistry: a situational analysis of staff and student perspectives: perspectives of plagiarism in dentistry. Eur J Dent Educ 16(1):e180-e186 doi:10.1111/j.1600-0579.2011.00695.x

Fuller JL, Killip DE (1979) Do dental students cheat? J Dent Educ 43(13):666-670

Graham BS, Knight GW, Graham L (2016) Dental student academic integrity in US dental schools: current status and recommendations for enhancement. J Dent Educ 80(1):5-13

Hutchins B, Cobb S (2008) When will we be ready for academic integrity? J Dent Educ 72(3):359-363

Koerber A, Botto RW, Pendleton DD, Albazzaz MB, Doshi SJ, Rinando VA (2005) Enhancing ethical behavior: views of students, administrators, and faculty. J Dent Educ 69(2):213-231

Koletsi-Kounari H, Polychronopoulou A, Reppa C, Teplitsky PE (2011) Penalties for academic dishonesty in a greek dental school environment. J Dent Educ 75(10):1383-1389

Kumar S, Dagli RJ, Mathur A, Jain M, Prabu D, Kulkarni S (2009) Perceived sources of stress amongst Indian dental students. Eur J Dent Educ 13(1):39-45. doi:10.1111/j.1600-0579.2008.00535.x

Kumwenda B, Dowell J, Husbands A (2013) Is embellishing UCAS personal statements accepted practice in applications to medicine and dentistry? Med Teach 35(7):599-603. doi:10.3109/0142159X.2013.798402

Lancaster DM, Gardiner JF, Strother EA, Boozer CH (1989) Dental students' class attitudes: a four year study. J Am Coll Dent 56(4):30-35

Lingen MW (2006) Tales of academic dishonesty and what do we do about it? Oral Surgery, Oral Medicine, Oral Pathology. Oral Radiol Endod 102(4):429-430. doi:10.1016/j.tripleo.2006.08.010

Muhney KA, Gutmann ME, Schneiderman E, DeWald JP, McCann A, Campbell PR (2008) The prevalence of academic dishonesty in Texas dental hygiene programs. J Dent Educ 72(11):1247-1260

National Dental Examining Board of Canada. (2017). Objective Structured Clinical Examination 2017 Protocol Retrieved from https://ndeb-bned.ca/sites/ndeb/files/pdf/Protocols/2017/osce_2017_protocol_-_revised_july_2017.pdf

Quick KK (2014) A humanistic environment for dental schools: what are dental students experiencing? J Dent Educ 78(12):1629-1635

Sierles F, Hendrickx I, Circle S (1980) Cheating in medical school. Acad Med 55(2):124-125

Teplitsky PE (2002) Perceptions of Canadian dental faculty and students about appropriate penalties for academic dishonesty. J Dent Educ 66(4):485-506

Turner SP, Beemsterboer PL (2003) Enhancing academic integrity: formulating effective honor codes. J Dent Educ 67(10): 1122

Warman E, Harvan RA, Weidman B (1994) Dental students' attitudes toward cheating. J Dent Educ 58(6):402-405

World Dental Schools List. Retrieved from http://www.dentaljuce.com/fruit/docs/World Dental schools.htm

Yip HK, Smales RJ (2000) Review of competency-based education in dentistry. Br Dent J 189(6):324-326. doi:10.1038/sj.bdj.4800758a

\section{Submit your manuscript to a SpringerOpen ${ }^{\circ}$ journal and benefit from:}

- Convenient online submission

- Rigorous peer review

Open access: articles freely available online

- High visibility within the field

- Retaining the copyright to your article

Submit your next manuscript at $\gg$ springeropen.com 